\title{
Designing of the Park-and-Ride Place between the Slow Traffic and Public Transport Integration
}

\author{
Zhuo Wang a, * \\ School of Traffic and Transportation, Dalian Institute of Science and Technology, Dalian 116052, \\ China \\ aichigo_715@163.com
}

Keywords: traffic planning, public transport, slow traffic, park-and-ride place.

\begin{abstract}
Experience suggests that urban developing both at home and abroad, in the past few decades, as the main body of the traffic development mode, motor vehicle traffic has many problems. The popularization of cars is in the change of people's life, at the same time, it also leads to the emergence of a series of urban problems, including tail gas pollution, traffic accidents, traffic congestion, etc. In order to alleviate or avoid these problems, many countries in the world and gradually started to 'encourage slow and limit car development' for the purpose of the comprehensive traffic development strategy. Slow traffic is mainly used to meet the demand of residents for short distance travelling, and to solve the problem of "the last kilometer" must change to the way, it is also an important part of urban transportation system. The linkage problem between the slow traffic and public traffic integration is an important indicator of green travel mode choice. Planning and designing park-and-ride place between the slow traffic and three kinds of public transportation, making the "Slow traffic + Public transport" transfer to parking facilities in the construction process have the theoretical basis, and can be used according to the concrete situation.
\end{abstract}

\section{Introduction}

At present, China is actively promoting the development of low-carbon transport, public transportation, rail transit construction and bicycle are in priority Green transportation has become the top priority of urban planning. Green transportation can be divided into two aspects: public transport and slow traffic. Public transport, namely security bus priority, bus transmission efficiency and service quality improvement, making residents' public transportation habits, and implementation of public transport motor travel no less than 80\%; Slow traffic is to build comfortable and pleasant space slowly, promote convenient safe interesting bicycle travel and walk [1]. Therefore, slow traffic and public transportation integration is the trend of the development of urban green traffic, "slow traffic and public transport" research question can significantly improve the convenience degree and transport efficiency of the residents between the two kinds of way, increase the proportion of green traffic.

\section{2. "Slow traffic + Public transport" Transfer Parking Designing}

Under the mode of "Slow traffic + public transport" integration, each public transportation site has strong aggregation effect, attracting the surrounding residents' slow traffic, and forms a reasonable transfer area, where passengers can walk or ride a bike to get public transport. Therefore, the public transport site area should consider the passenger travel time, travel distance, and physical activity [2]. In the bicycle and the linkage between the public transport system, reasonable transfer to transfer facilities, bicycle and slow traffic cohesion to the evaluation of public transportation is an important content, and mainly composed of three parts, as shown in Fig. 1. 


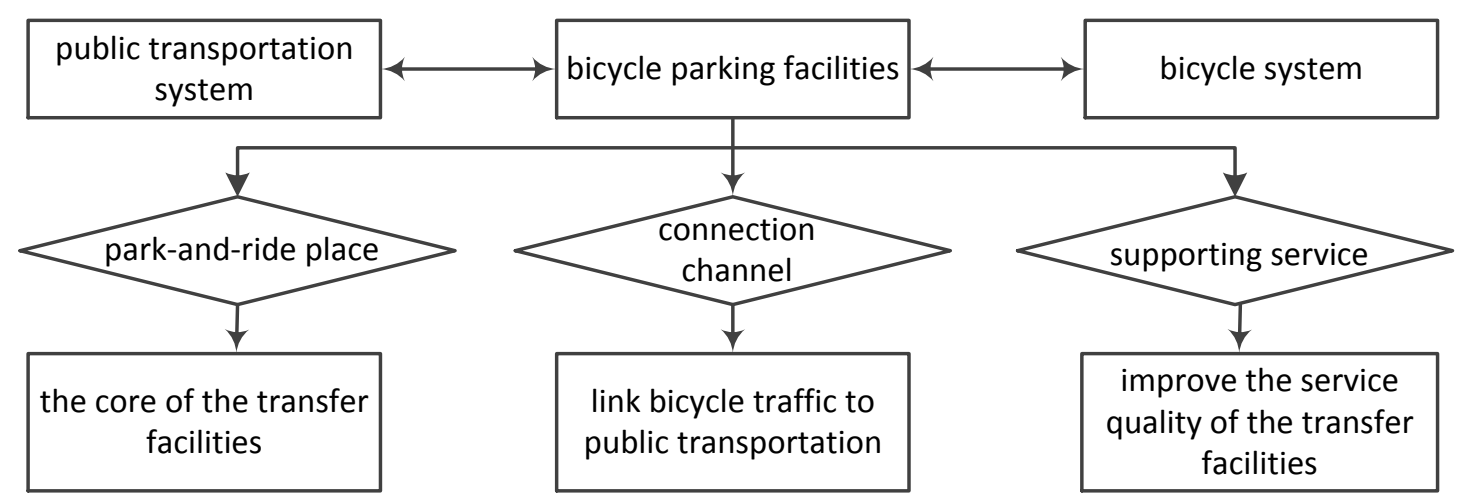

Fig. 1 Flow chart of bicycle transfer system

\section{Design for the Interchange Parking Lot between Slow Traffic and Conventional Public Transport}

\subsection{Location selection}

The bike parking lot at the transfer point should be located near the bus stop, so that residents can walk to the bus stop. But in order to reduce the traffic conflict between the bus and bicycle, the parking lot should be set up to meet the following three requirements [3]:

Security. Try to avoid the interference to the road motor vehicles and buses run in and out of the bus stops, it should not occupy too much space for waiting in order to ensure the space demand of the people waiting for the bus.

Convenience. To ensure that the bicycle parking lot to the two directions of bus stops has convenient walkways .Make it safe and easy to walk on bus stops and parking lots.

Consider the bicycle. Keep the bicycle safe and convenient.

\subsection{Determine the Number of Bicycles for Transfer Points}

The accuracy of the measurement of the vehicle is directly affected by the area of the parking lot, which can affect the function of the parking lot. The impact factors of the vehicle inventory determination are the following [4]:

The proportion of long distance travelling. Generally when the travelling distance of more than 7 $\mathrm{km}$ accounts for $35.5 \%$ of travel, and the distance of the ride is roughly in half an hour or more, it is no longer suitable for simple bicycle travelling, and travelers should consider change to the bus.

Range of transfer attraction. In general, the shortest distance of the bicycle travelling is about $1 \mathrm{~km}$, and the travel time is about 5 mins. The maximum appeal should not exceed the average travel distance of the current bicycle, which is about $5.5 \mathrm{~km}$.

Population composition proportion. The elderly and children are not suitable for bicycle travelling, the primary and middle school students in general study in a neighborhood school, making transfer proportion not high, so the highest proportion of transfer in general is for adult workers.

The proportion of traffic volume of bus stops. It refers to the proportion of point of transfer to bus station capacity of services within the scope of all bus stops, in general, the bigger bus stop is, the greater the dock is, and the traffic nature also is bigger, has been attracting more transfer quantity. It is difficult to determine the time of parking and the factors such as the turnover rate, which is not shown here.

The average turnover of bicycle. It refers to the turns of bicycle parking in the parking lot. Generally adult workers working go out early and come home late, only stopping the bike once a day on average, so the turnover rate of 1 . Thus, the formula for calculating the amount of bicycle storage for the transfer point is as follows:

$V=(K \times L \times P \times A) / H$

Formula:

$K$ - the percentage of long-distance trips, $\%$;

$L$ - the proportion of bikes in the service range from where you live, $\%$; 
$P$ - the number of adult workers in the range of attraction, number of people;

$A$ - the proportion of traffic on the bus site, $\%$;

$H$ - the average turnover rate of bicycle, frequency.

\subsection{Parking Design in the Parking Lot}

The bicycle can be divided into 4 types of two-way vertical, single-row vertical, double row oblique type and single row of ram in the vicinity of the transfer point area as a bicycle parking lot, according to the relationship between vehicles and the channel. The parking width reference dimensions were vertical $0.6 \mathrm{~m}$, oblique type $0.5 \mathrm{~m}$. The angle of the vehicle and the channel in the skew is generally divided into $30^{\circ}, 45^{\circ}$ and $60^{\circ}$, as shown in Fig2.
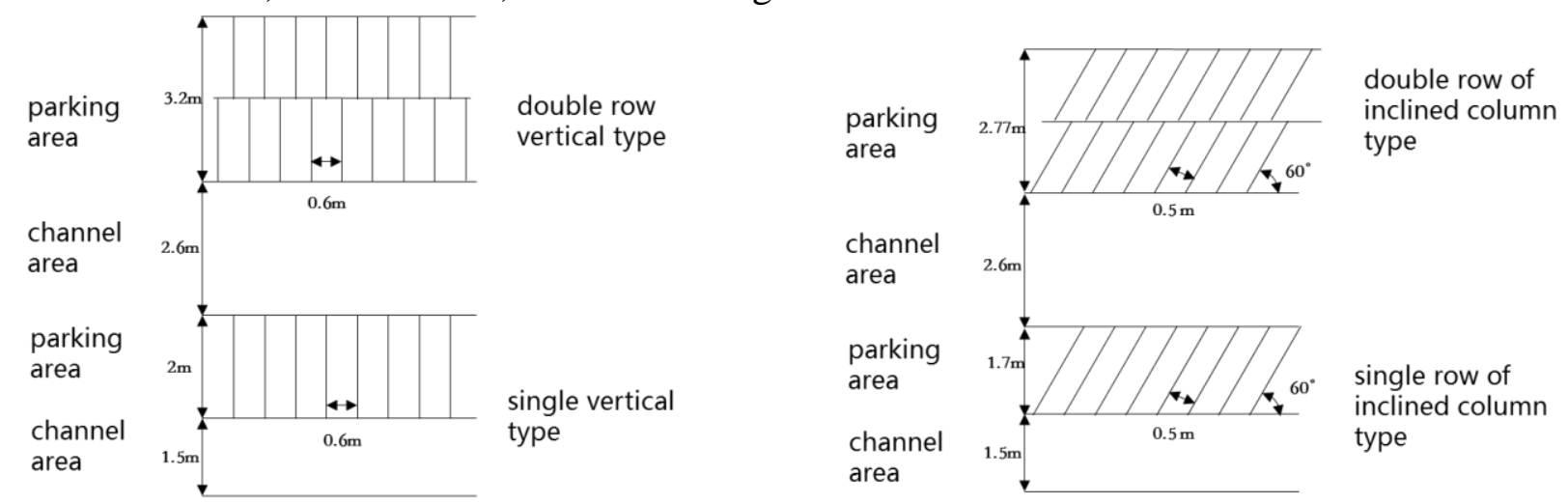

Fig. 2 Design drawings for parking pattern in a parking lot

\section{Design for the Interchange Parking Lot between Slow Traffic and BRT}

\subsection{Location Selection}

In the bicycle transfer to BRT design, first it should adhere to the "near layout" and "decentralized planning" basic principles, and then taking into account the BRT line is mainly concentrated in the central city, thus considering the "land intensive" and "landscape coordination".

BRT parking lot can be divided into the original and terminal stations parking and midway stations parking in accordance with its specific location on the whole line. The original and terminal stations are usually distributed in the edge of the city, mainly provide transfer service for the long distance travel of the bicycle, connecting the periphery and the city center, making the size of the bike parking lot large, it is necessary to ensure that passengers after the transfer can get into the fast bus station as soon as possible, and also ensure a special import and export directly connected to the bus station to avoid bypass. While the Midway transfer car park is based on the layout of the line, only serve in the small area around the site, bicycle transfer volume is relatively small. The comparatively mature BRT are basically using the central lane, setting up passengers' dedicated walking channel in the middle of the station, which also requires taking into account. Considering the limited number of passengers in the midway transfer and the source is more dispersed, it should make full use of all directions in the direction of the cross-street facilities in the vicinity of the open space, each transfer point of the parking scale is not too large, at the same time shorten the passengers of the bypass distance.

\subsection{Determine the Amount of Bicycle Storage of theTransfer Point}

The calculation of bicycles used to transfer to the BRT point can refer to the slow transit and conventional bus transfer car park formulas, only the values of the indicators are different. In terms of population composition, bicycle transfer to BRT population will be slightly different from that to take the conventional bus. The people selected the bicycle transfer to BRT generally have a low income and long travel distance, age concentrated in the 15 to 55 years old, in which the majority are still office workers. As for the parking lot design and the former is roughly the same as the former. 


\section{Design For The Interchange Parking Lot Between Slow Traffic and Rail Transit}

\subsection{Location Selection}

Bicycle and rail transit transfer connection is still through the interchange transfer to achieve, the location of the parking lot should be adhering to the "safe, convenient and efficient" principle, and also consider the factors such as the main flow of bicycles, passengers transfer distance and the surrounding conventional bus station to connect. According to the parking lot and the rail transit station connection mode, the parking lot location selection can be divided into four kinds: (1) the parking lot set in the rail transit station near the entrance side of the road, which is cheap and easy to manage; (2) parking lot set under the viaduct, which can save land; (3) the bike parking lot and underground station hall set up directly to the use of station underground hall floor which can save the land, shorten the transfer distance, and the environment is better, but the entrance with a slope requires higher design level and the cost is also high; (4) bike parking lot set in the station before the traffic square, then the bike parking lot should be avoided the motor vehicle connection facilities mixed arrangement, try to reduce the conflict between bike flow and motor vehicle flow.

\subsection{Determine the Amount of Bicycle Storage for the Transfer Point}

The influence factors of bicycle storage on bicycle and BRT transfer points, is similar to the influence factors of the slow traffic and the conventional bus changing bike parking lot. These include long-distance travel, transfer and the proportion of the population. The formula for calculating bike storage for transfer points is as follows:

$$
V=(K \times L \times P) / H
$$

Formula:

$K$ - the percentage of long-distance trips, \%;

$L-$ the proportion of bikes in the service range from where you live, $\%$;

$P$ - to attract the number of adult workers in the range, number of people;

$H$ - the average turnover rate for bicycles, frequency.

The transfer attraction here is defined as the center of the rail transit site, a circular range of $3.6 \mathrm{~km}$ radius. The bicycle and rail transit parking lot is mainly used in planar, three-dimensional ram-type and mechanical type, with maximum utilization of existing land.

\section{Conclusion}

Slow traffic is an important addition to urban transportation. The integration of slow traffic and public transportation is the top priority of the interchange. The two are reasonable to join and complement each other to form a harmonious and integrated network. It can fundamentally make the residents to choose green travel way, gradually reduce the use of motor transport in order to guide resident's private cars to public transportation. Closely integrated by walking, cycling and public transport system, the construction of the integration of the transfer of the parking lot, making the travelers to take public transit can complete seamless to address the critical issue of "last mile" and achieve the goal of guiding "walking + bus" and "bicycle + bus", reducing car collisions. It will improve the service level of the public transport system and the city bus system.

\section{References}

[1]. Wei Yu, Qian, et al., etc., the planning and design of the transportation system in Hangzhou [J]. Urban Transportation, (2009) 2. 44-56.

[2]. Peng Hu, Based on the slow traffic integration research [D]. Huazhong University of Science and Technology. Wuhan. (2008)

[3]. Qiuping Wang, Ni Luo, The location of the parking facilities for bicycle and public transport [J]. Transportation Technology and Economy. (2013)15 (6) .14-17 
[4]. Hua Peng, Jingtao Ma, Lijun Sun, The planning and research of bicycle transportation and rapid transit system. [J]. Planners, (2010) 26. 84-87. 